\title{
A Quantitative View on Naturally Occurring Autoantibodies in Neuro- degenerative Diseases
}

Yannick Kronimus, Richard Dodel*, Sascha Neumann

Department of Geriatrics, University Duisburg-Essen, Germaniastrasse 1-3, 45356 Essen, Germany

\section{Article Info}

\section{Article Notes}

Received: May 28, 2018

Accepted: July 05, 2018

\section{*Correspondence:}

Dr. Richard Dodel, MD, Department of Geriatrics, University

Duisburg-Essen, Germaniastrasse 1-3, 45356 Essen, Germany; Telephone No: +49 (0)201-89760; Fax No: +49 (0)201 8976229; E-mail: richard.dodel@uk-essen.de.

(c) 2018 Dodel R. This article is distributed under the terms of the Creative Commons Attribution 4.0 International License

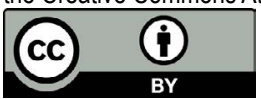

\section{Keywords:}

Naturally occurring autoantibodies

Alzheimer's disease

Parkinson's disease

Serum titers

CSF titers

Beta-Amyloid; Alpha-Synuclein

ELISA; Antibody

Antibodies

\begin{abstract}
Accumulation and aggregation of Beta-Amyloid $(A \beta)$ and Alpha-Synuclein ( $\alpha$-Syn) are considered as central or even causative for the development of Alzheimer's (AD) and Parkinson's disease (PD). Therefore, the regulation of these proteins seems to be an essential aspect for prevention and is of central interest in current research aiming to find therapeutic approaches. The human immunological repertoire already contains such a regulatory system. Naturally occurring autoantibodies (nAbs) against the proteins $A \beta$ (nAbs-A $\beta$ ) and $\alpha$-Syn (nAbs- $\alpha$-Syn) are part of the innate immune system and modulate the metabolism of their specific antigens including protein clearance and inhibition of aggregation. Thus, many researchers hypothesize that in the course of $A D$ and PD, quantitative alterations of $n A b s-A \beta$ and $n A b s-\alpha-S y n$ arise resulting in impaired proteastasis. Such alterations would represent promising, reliable biomarkers and indicate potential approaches for therapeutic strategies. Hence, it is not surprising that many studies dealing with $n A b s-A \beta$ and $n A b s-\alpha-S y n$ titers in AD and PD patients in comparison to control participants are available in the literature. In this mini review, we summarize the current evidence. Furthermore, we critically discuss problems and future requirements for nAbs quantification when a clinical application is the overriding goal.
\end{abstract}

\section{Introduction}

The human antibody repertoire can be subdivided into conventional and naturally occurring antibodies, based on their originating B cell type. While conventional antibodies derive during life from plasma and memory B cells differentiated from B2 or marginal zone B cells after antigenic activation, naturally occurring antibodies arise in fetogenetic periods and thus are present from birth ${ }^{1-4}$. They are produced from $B 1$ cells without extrinsic stimuli and $\mathrm{T}$ cell assistance. A special characteristic of B1 cells is the germline-closed configuration of the recombined immunoglobulin genes resulting in less hypermutated and affinity matured antibodies with lower affinities ${ }^{5}$. Although conventional antibodies are usually the main subject in textbooks, naturally occurring antibodies account for the greatest portion in humans ${ }^{6}$. Among them, a fraction shows autoreactivity and is termed naturally occurring autoantibodies (nAbs), which are mostly from the IgM and IgA, fewer from the IgG type $\mathrm{e}^{7}$. They circulate through human body fluids, maintain physiological homeostasis, support the clearance of distinct secreted proteins and apoptotic cells, and protect from pathologically altered structures like oxidatively damaged, aggregated, and non-functional lipids and proteins ${ }^{8,9}$. In the following sections we want to focus on the beneficial aspects of nAbs and their potential clinical applications, especially for therapeutics and diagnostics in neurodegenerative disorders. 


\section{Clinical Potential of AD and PD Associated nAbs}

The two most common neurodegenerative disorders - Alzheimer's (AD) and Parkinson's disease (PD) - are among others histopathologically characterized by extra- and intracytoplasmic protein deposits primarily consisting of the proteins beta-amyloid $(A \beta)$ in the case of $\mathrm{AD}$ or alpha-synuclein ( $\alpha$-Syn) in the case of $\mathrm{PD}^{10,11}$. Albeit the accurate pathomechanism has not been fully clarified, neither for AD nor for PD, it is widely accepted that the metabolic dysregulation of the proteins has etiological significance ${ }^{12}$. For $A \beta$, especially the isoform containing 42 amino acids $\left(A \beta_{42}\right)$ has been identified as minatory as it exhibits heightened hydrophobicity. The accumulation and aggregation of both, $\mathrm{A} \beta$ and $\alpha$-Syn into small soluble oligomers and fibrils have been connected to many (in)direct neuro- and cytotoxic effects indicating their participation in disease onset and progression ${ }^{13-16}$. Furthermore, especially for $\alpha$-Syn, a transmitting pathological mechanism is highly hypothesized within literature encompassing the spreading of aggregates from cell to cell ${ }^{17}$.

Although AD- and PD-associated protein deposition and the resulting detrimental effects are more obvious in the central nervous system (CNS), A $\beta$ as well as $\alpha$-Syn dysregulation also occurs in the periphery. In particular, this is indicated by vascular $A \beta$ deposition - very common in $\mathrm{AD}$ - as well as $\alpha$-Syn pathology detectable in erythrocytes and nerve cells of the enteric system in PD patients ${ }^{18-20}$.

Thus, since many years and considering all aspects, researchers follow the idea of an early and preventive intervention counteracting protein dysregulation, aggregation, and propagation as it is believed to modify disease progression in AD, PD, and other proteinopathies. Here, $A \beta_{42}$ and/or $\alpha$-Syn specific antibody treatment could represent a promising therapeutic strategy due to a targeted modulation of the metabolism of their antigen. Such approaches have already been tested in diverse in-vitro and in-vivo experiments and revealed positive effects including reduced protein deposits and decreased neurodegeneration, however, failed in phase three at the latest when tested in the clinical state of $\mathrm{AD}^{21-25}$.

It is an interesting fact that antibodies that maintain tissue homeostasis and proteostasis are already present in humans ${ }^{26,27}$. In child- and adulthood as well as in health and disease, nAbs recognizing the proteins $A \beta_{42}$ (nAbs$\mathrm{A} \beta_{42}$ ) and $\alpha$-Syn (nAbs- $\alpha$-Syn) but also further proteins that become misfolded in disease cases (such as prion and tau protein) are detectable in the serum and cerebrospinal fluid (CSF) suggesting the presence of innate protective mechanisms ${ }^{2,27-32}$. Quantitative or qualitative alterations of nAbs could impair their normal functions, worsen the protection system, and thus represent causal or supporting factors for the development of $\mathrm{AD}$ and PD. Especially quantitative alterations, namely changed titers, may act as biomarkers in clinical applications and illustrate restoring of $A \beta_{42}$ and $\alpha$-Syn specific antibodies as a promising therapeutic strategy. It is not surprising that a number of studies investigating titers of nAbs- $\mathrm{A} \beta_{42} / \alpha$-Syn have already been published in the past and will be following discussed.

\section{nAbs-A $\beta$ titers}

A large body of literature exists harboring inconsistent, even contrasting data regarding nAbs- $A \beta$ titers in $A D$ patients. In a comprehensive study by Britschgi et al. (2009), nAbs- $A \beta$ recognizing various natures of its antigen could be detected within serum and CSF samples of AD patients and nondemented control subjects of different ages $^{33}$. Using ELISA technique and antigen microarrays the highest reactivity was uncovered for aggregated and posttranslationally modified $A \beta_{40}$ and $A \beta_{42}$ forms. Between AD patients and controls, no significant differences of nAbs- $\mathrm{A} \beta_{42}$ plasma titers against oligomeric or monomeric protein forms were identified. However, comparing mildly to moderately and severely affected AD patients, the latter group showed in one sample set significantly decreased nAbs- $A \beta_{42}$ plasma titers suggesting a role in disease progression. Unchanged nAbs-A $\beta$ titers in $A D$ patients were also verified in further studies examining $n A b s-A \beta_{40}$ and $A \beta_{42}{ }^{26,28}$. Marcello and coworkers (2011) revealed similar results even the experimental setup was limited to IgM autoantibodies ${ }^{34}$.

Although they were not able to detect altered nAbs$\mathrm{A} \beta_{42}$ titers, plasma level of nAbs recognizing $\mathrm{N}$-truncated $A \beta$ consisting of the amino acid sequence three to seven and a modified pyroglutamate $\left({ }_{\mathrm{pGlu}} \mathrm{A} \beta_{3-7}\right)$ was significantly decreased in patients suffering from AD. Such peptides have often been detected in the brains of AD patients and were shown to strengthen the aggregation process ${ }^{35}$. Three further studies identified significantly changed blood titers of nAbs against distinct $A \beta$ forms using ELISA techniques. Qu et al. (2014) demonstrated reduced serum titers of nAbs recognizing $A \beta_{1-15}$ but unchanged nAbs- $A \beta$ against soluble and aggregated full-length peptides in AD patients ${ }^{36}$. Moir and colleagues (2005) found decreased nAbs levels against redox-modified $A \beta_{40}$ and again unchanged nAbs- $A \beta_{40}$ in AD patients ${ }^{37}$. In contrast, Gruden and coworkers (2007) detected significantly increased nAbs portions in female $\mathrm{AD}$ patients, namely autoantibodies against oligomeric $A \beta$ consisting of the amino acids 25 to $35^{38}$. This peptide fragment - inter alia detectable in AD brains - is often used as a model system as it maintains the neurotoxic properties of $A \beta$ with the simultaneous option of better controlling the aggregation process ${ }^{39}$. Elevated titers of unbound autoantibodies recognizing these eleven amino acids containing and most toxic sequence may be a hint 
for decreased but necessary antigen binding and thus regulation of $A \beta_{25-35}$ what may result in disease onset or progression. The exclusive investigation of such antibodies has the ability to be a critical step and advantage as all further $\mathrm{A} \beta$ targeting $\mathrm{nAbs}$ with various epitopes could overlap a specific effect of the indeed crucial sequence.

As mentioned above, there are also contrary data available including studies that revealed increased or decreased $n A b s$ portions recognizing the full-length peptide $\mathrm{A} \beta_{42}$. On the one hand, some ELISA as well as radiolabeled immuno-precipitation based experiments have uncovered lowered nAbs- $A \beta_{42}$ serum titers in $A D$ patients when compared to healthy or cognitively unremarkable subjects $^{40,41}$. On the other hand, Nath and colleagues (2003) determined elevated nAbs serum titers against soluble and pre-aggregated $A \beta_{42}$ in $A D$ patients ${ }^{42}$. In the same study, CSF samples have also been investigated, however, only three of the AD patients - but interestingly the severely affected ones - and none of the controls contained detectable amounts of $n A b s-A \beta_{42}$. Since CSF data are generally less frequent than serum data -it encompasses a more invasive clinical method - only a few more studies dealing with variable nAbs structures have been published. For example, $\mathrm{Du}$ and colleagues (2001) revealed decreased nAbs- $\mathrm{A} \beta_{40}$ titers in CSF of AD patients using ELISA ${ }^{43}$. Within a second study published by Maftei et al. (2013) the same method was applied to analyze $n A b s / A \beta_{42}$ immune complexes. Here, a significantly increased portion in the CSF of people suffering from AD was detected ${ }^{44}$. Although the studies investigated nAbs targeting different antigenic structures, similar effects can underlie both observations as decreased free nAbs titers can be the result of increased $A \beta$ bound nAbs (immune complexes).

\section{nAbs- $\alpha-S y n$ titers}

Conflicting results of nAbs- $\alpha$-Syn titers are also available in literature about PD. Two exemplary studies using ELISA technique and dealing with serum and CSF nAbs- $\alpha$-Syn demonstrate significantly elevated autoantibody titers in patients suffering from $\mathrm{PD}^{45,46}$. While Horvath and colleagues (2017) found differences between PD patients and control subjects in both, CSF and serum, Akhtar and coworkers (2018) detected only increased nAbs levels in the CSF. This is of particular interest as Horvath revealed a stronger effect size for changed serum titers and the loss of significant differences of CSF titers when separating the patient cohort into mild and moderate affected individuals. In line with these observations, Gruden et al. (2011) as well as Yanamandra et al. (2011) performed serum ELISA experiments and surface plasmon resonance spectroscopy with monomeric and aggregated $\alpha$-Syn, identified increased nAbs- $\alpha$-Syn titers against both protein forms in PD

Table 1. NAbs Serum/Plasma and CSF titers in AD patients compared to control subjects. Summary of exemplified studies investigating changed serum/plasma and CSF concentration of nAbs-A $\beta$. For each study and if information were available ( $\mathrm{n}$.a. $=$ not available), $p$-value of the statistical analysis, age- (AM), gender-matching (GM), and Mini-Mental-State-Examination Score of the study participants as well as the used method are shown. *No comparison possible as nAbs-AB42 were only detectable in the CSF of severely affected AD patients.

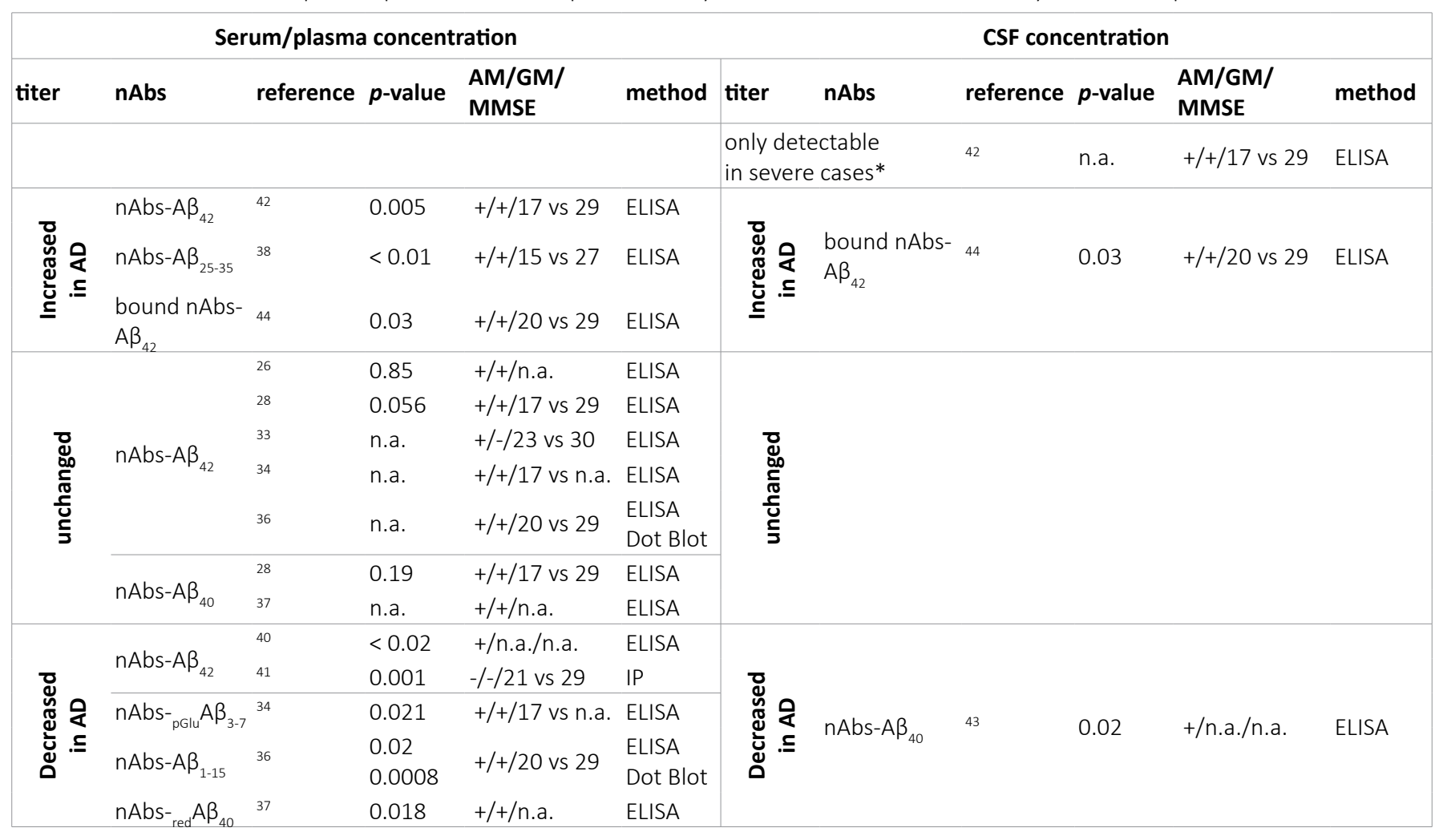


Table 2. NAbs Serum/Plasma and CSF titers in PD patients compared to control subjects. Summary of exemplified studies investigating changed serum/plasma and CSF concentration of nAbs- $\alpha$-Syn. For each study and if information were available (n.a. = not available), $p$-value of the statistical analysis, age- (AM), gender-matching (GM), and Hoehn \& Yahr Scale (H\&Y; median or *mean) of the study participants as well as the used method are shown.

\begin{tabular}{|c|c|c|c|c|c|c|c|c|c|c|c|}
\hline \multicolumn{6}{|c|}{ Serum/plasma concentration } & \multicolumn{6}{|c|}{ CSF concentration } \\
\hline titer & nAbs & reference & $p$-value & $\begin{array}{l}\text { AM/GM/ } \\
\text { H\&Y* }\end{array}$ & method & titer & nAbs & reference & $p$-value & $\begin{array}{l}\text { AM/GM/ } \\
\text { H\&Y* }\end{array}$ & method \\
\hline \multirow{5}{*}{ 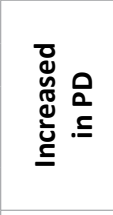 } & \multirow{5}{*}{ nAbs- $\alpha-S y n$} & 46 & $<0.05$ & n.a./+/2 & ELISA & \multirow{5}{*}{ 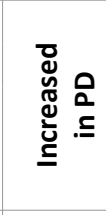 } & \multirow{5}{*}{ nAbs- $\alpha-S y n$} & \multirow{5}{*}{45} & \multirow{3}{*}{0.016} & \multirow{3}{*}{$+/-/ 3$} & \multirow{2}{*}{ ELISA } \\
\hline & & 47 & $<0.01$ & $+/+/ 2.1^{*}$ & ELISA & & & & & & \\
\hline & & \multirow[t]{2}{*}{48} & $<0.007$ & n.a. & ELISA/WB & & & & & & \multirow{3}{*}{ ELISA } \\
\hline & & & $<0.001$ & $11 . a$. & SPR & & & & $<0.05$ & n.a./+/2 & \\
\hline & & 49 & $<0.001$ & $+/+/ 1$ & ELISA & & & & & & \\
\hline \multirow{4}{*}{ 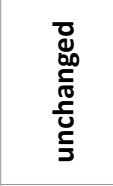 } & \multirow{4}{*}{ nAbs- $\alpha-S y n$} & 45 & 0.19 & $+/+/ 3$ & ELISA & \multirow{4}{*}{ 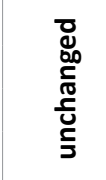 } & \multirow{4}{*}{ nAbs- $\alpha-S y n$} & & & & \\
\hline & & 51 & 0.5 & $-/-/ 2$ & ELISA & & & & & & \\
\hline & & 52 & 0.69 & $+/+/ 1.3^{*}$ & ELISA & & & & & & \\
\hline & & 53 & n.a. & $-/-/ 2.4$ & ELISA/WB & & & & & & \\
\hline \multirow{3}{*}{ 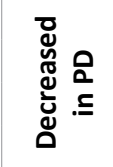 } & \multirow{2}{*}{ nAbs- $\alpha-S y n$} & 30 & $<0.05$ & $+/+/ 2$ & ELISA & \multirow{3}{*}{ 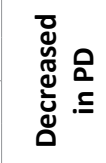 } & \multirow{3}{*}{ nAbs- $\alpha-S y n$} & & & & \\
\hline & & 50 & 0.005 & $-/-/ 2$ & ELISA & & & & & & \\
\hline & $\begin{array}{l}\text { bound nAbs- } \\
\alpha-S y n\end{array}$ & 50 & 0.042 & $-/-/ 2$ & ELISA & & & & & & \\
\hline
\end{tabular}

patients, and revealed an attenuated effect with increasing disease duration ${ }^{47,48}$. In a further publication, Shalash and colleagues (2017) expanded the comparison of nAbs- $\alpha$-Syn serum levels to PD patients, controls, and AD patients and again detected an elevated portion in the PD cohort ${ }^{49}$.

These studies provide the impression of increased nAbs- $\alpha$-Syn titers in especially early stages of PD suggesting disease duration as a critical factor for such findings. However, contrasting hints with both, short- and longterm affected PD patients and correspondent differences in age and disease severity exist as well. Besong-Agbo et al. (2013) as well as Brudek et al. (2017) demonstrated significantly decreased nAbs- $\alpha$-Syn serum titers in the plasma and serum of PD patients ${ }^{30,50}$. Additionally, in the latter paper PD patients were also shown to exhibit lower titers of $\alpha$-Syn/nAbs immune complexes resulting in a decreased total nAbs- $\alpha$-Syn fraction.

As opposed to the already outlined studies, Maetzler and coworkers published in 2014 the presence of comparable nAbs- $\alpha$-Syn serum levels in PD and healthy persons ${ }^{51}$. Such unchanged nAbs- $\alpha$-Syn titers have also been confirmed in two further publications investigating serum or plasma samples of PD patients and healthy controls ${ }^{52,53}$. Here, especially the publication by Smith et al. (2012) is of great interest as they additionally summarize and demonstrate conflicting studies regarding $\alpha$-Syn protein ${ }^{52}$.

\section{Conclusion and Future Perspectives}

For the development of reliable diagnostic markers but also as therapeutic targets, the determination of nAbs-A $\beta$ and nAbs- $\alpha$-Syn titers are of certain interest in AD and PD research. However, as mentioned above, in both cases many different and contrasting results have been published in the last years including increased, decreased, and unchanged nAbs levels in affected persons. Not until this drawback is eliminated, the application of nAbs titers has a chance to receive clinical significance.

A number of reasons might be responsible for the great variance and contrary data of altered nAbs titer in $A D$ and PD. In many cases, typical parameters like incorrect diagnoses of the included participants, small sample sizes and technical limitations are cited as causes. However, it is largely impossible that the contrasting findings are a methodbased effect as in almost all cases ELISA technique was applied to determine nAbs titers. Within the experimental setups, differences in the sample preparation are more likely responsible for contrary results. The utilization of different body fluids including plasma, serum, or CSF harbors also risks for fluctuations like a previous antibody purification. Additionally, a number of further variabilities may account for inconsistent data on $n A b s-A \beta$ and $n A b s-\alpha-$ Syn titers. Here, two main factors represent 1) the choice of the incorporated participants and 2) the antigen that is used to determine its corresponding nAbs titer. Control probands often range from younger healthy controls to agematched patients without disease-associated symptoms ${ }^{36,51}$. Furthermore, patient cohorts often differ in disease severity and duration. Such variabilities result in different physical and immunological conditions and may significantly influence nAbs titer analyses. Especially, the latter aspect is of utmost importance as directly age dependent and indirectly medication mediated effects on antibody titers have been identified ${ }^{54-56}$. This should not be disregarded even though authors including individuals without agematching usually argue that their cohorts do not show positive correlation between age and antibody titers. 
Different protein states can also greatly influence results of ELISA and related assays. The choice between recombinant or synthetic peptides including the expression system with its particular posttranslational modifications (PTMs), full-length or peptide fragments, and monomeric or oligomeric structures probably impact antigen binding. Regarding the last aspect, especially nAbs- $A \beta$ analyses exhibit great potential for variability as $A \beta$ shows a naturally high aggregation property that complicates the control of its exact state e.g. during the ELISA coating process ${ }^{57}$.

Additionally, most of the performed studies feature limitation in their experimental setup in general as their analyses are restricted to either the antigen bound or the unbound nAbs fraction. However, to draw a comprehensive picture about the immunological status in $\mathrm{AD}$ and $\mathrm{PD}$, information about both conditions as well as the antigen concentration are important to be considered.

Overall, for the clinical application of quantitative analyses of nAbs-A $\beta$ and $n A b s-\alpha-S y n$, the primary future goal has to be the elimination of the already described major drawbacks within the experimental setups. This includes both, the possible causes of failure and variability as well as the consideration of antigen/nAbs immune complexes and free circulating nAbs. Here, a standardization of the experimental setup with the formulation of guidelines within a consortium would be helpful to decreased variabilities across studies and laboratories. A similar procedure has been applied for biomarkers in $\mathrm{AD}$, called Global Biomarker Standardization Consortium (GBSC) ${ }^{58}$.

If nAbs-A $\beta$ and nAbs- $\alpha$-Syn quantification will be reliably and reproducibly executed in future studies, thresholds for concentration can be set for its application as a diagnostic biomarker provided that actual differences between healthy and diseased people are present. Furthermore, such findings would demonstrate the necessity of an antibody targeted therapy. On the other hand, quantitative differences do not represent the only possible diseasecausing alteration as especially qualitative properties like avidity, immune activation, or PTMs are also crucial properties of nAbs.

\section{References}

1. LeBien TW,Tedder TF. B lymphocytes: how they develop and function. Blood. 2008, 112: 1570-1580

2. Gold M, Pul R, Bach JP, et al. Pathogenic and physiological autoantibodies in the central nervous system. Immunol Rev. 2012, 248: 68-86

3. Griffin DO, Holodick NE,Rothstein TL. Human B1 cells in umbilical cord and adult peripheral blood express the novel phenotype CD20+ CD27+ CD43+ CD70. J Exp Med. 2011; 208: 67-80

4. Madi A, Bransburg-Zabary S, Kenett DY, et al. The natural autoantibody repertoire in newborns and adults: a current overview. Adv Exp Med Biol. 2012; 750: 198-212
5. Rothstein TL, Griffin DO, Holodick NE, et al. Human B-1 cells take the stage. Ann N Y Acad Sci. 2013; 1285: 97-114

6. Lutz HU. Naturally Occurring Antibodies (NAbs). Springer New York. 2012.

7. Shoenfeld Y, Meroni PL,Gershwin ME. Autoantibodies. Elsevier. 2014.

8. Lutz HU. Homeostatic roles of naturally occurring antibodies: an overview. J Autoimmun. 2007; 29: 287-294

9. Lutz HU, Binder CJ,Kaveri S. Naturally occurring autoantibodies in homeostasis and disease. Trends Immunol. 2009; 30: 43-51

10. Selkoe DJ,Hardy J. The amyloid hypothesis of Alzheimer's disease at 25 years. EMBO Mol Med. 2016; 8: 595-608

11. Davie CA. A review of Parkinson's disease. Br Med Bull. 2008; 86: $109-127$

12. Kikuchi K, Kidana K, Tatebe T, et al. Dysregulated Metabolism of the Amyloid-beta Protein and Therapeutic Approaches in Alzheimer Disease. J Cell Biochem. 2017; 118: 4183-4190

13. Brouillette J, Caillierez R, Zommer N, et al. Neurotoxicity and memory deficits induced by soluble low-molecular-weight amyloid-beta1-42 oligomers are revealed in vivo by using a novel animal model. J Neurosci. 2012; 32: 7852-7861

14. Celej MS, Sarroukh R, Goormaghtigh E, et al. Toxic prefibrillar alpha-synuclein amyloid oligomers adopt a distinctive antiparallel beta-sheet structure. Biochem J. 2012; 443: 719726

15. Danzer KM, Haasen D, Karow AR, et al. Different species of alpha-synuclein oligomers induce calcium influx and seeding. J Neurosci. 2007; 27: 9220-9232

16. Kayed R, Lasagna-Reeves CA. Molecular mechanisms of amyloid oligomers toxicity. J Alzheimers Dis. 2013; 33 Suppl 1: $567-78$

17. Recasens A, Dehay B. Alpha-synuclein spreading in Parkinson's disease. Front Neuroanat. 2014; 8: 159

18. Smith EE, Greenberg SM. Beta-amyloid, blood vessels, and brain function. Stroke. 2009; 40: 2601-2606

19. Savica R, Dyer RB, Mielke MM, et al. alpha-Synuclein in red blood cells is a potential diagnostic biomarker for Parkinson's disease. Movement Disorders. 2014; 29: S28-S30

20. Braak H, de Vos RAI, Bohl J, et al. Gastric alpha-synuclein immunoreactive inclusions in Meissner's and Auerbach's plexuses in cases staged for Parkinson's disease-related brain pathology. Neuroscience Letters. 2006; 396: 67-72

21. Hock C, Konietzko U, Streffer JR, et al. Antibodies against beta-amyloid slow cognitive decline in Alzheimer's disease. Neuron. 2003; 38: 547-554

22. Hartman RE, Izumi $\mathrm{Y}$, Bales $\mathrm{KR}$, et al. Treatment with an amyloid-beta antibody ameliorates plaque load, learning deficits, and hippocampal long-term potentiation in a mouse model of Alzheimer's disease. J Neurosci. 2005; 25: 62136220

23. Vandenberghe R, Rinne JO, Boada M, et al. Bapineuzumab for mild to moderate Alzheimer's disease in two global, randomized, phase 3 trials. Alzheimers Res Ther. 2016; 8: 18 
24. Shahaduzzaman M, Nash K, Hudson C, et al. Anti-human alpha-synuclein $\mathrm{N}$-terminal peptide antibody protects against dopaminergic cell death and ameliorates behavioral deficits in an AAV-alpha-synuclein rat model of Parkinson's disease. PLoS One. 2015; 10: e0116841

25. Schenk DB, Koller M, Ness DK, et al. First-in-human assessment of PRX002, an anti-alpha-synuclein monoclonal antibody, in healthy volunteers. Mov Disord. 2017; 32: 211-218

26. Hyman BT, Smith C, Buldyrev I, et al. Autoantibodies to amyloid-beta and Alzheimer's disease. Ann Neurol. 2001; 49: 808-810

27. Papachroni KK, Ninkina N, Papapanagiotou A, et al. Autoantibodies to alpha-synuclein in inherited Parkinson's disease. J Neurochem. 2007; 101: 749-756

28. Baril L, Nicolas L, Croisile B, et al. Immune response to Abetapeptides in peripheral blood from patients with Alzheimer's disease and control subjects. Neurosci Lett. 2004; 355: 226230

29. Kuhn I, Rogosch T, Schindler TI, et al. Serum titers of autoantibodies against alpha-synuclein and tau in child- and adulthood. J Neuroimmunol. 2018; 315: 33-39

30. Besong-Agbo D, Wolf E, Jessen F, et al. Naturally occurring alpha-synuclein autoantibody levels are lower in patients with Parkinson disease. Neurology. 2013; 80: 169-175

31. Kronimus Y, Albus A, Balzer-Geldsetzer M, et al. Naturally Occurring Autoantibodies against Tau Protein Are Reduced in Parkinson's Disease Dementia. PLoS One. 2016; 11: e0164953

32. Wei X, Roettger Y, Tan B, et al. Human anti-prion antibodies block prion peptide fibril formation and neurotoxicity. J Biol Chem. 2012; 287: 12858-12866

33. Britschgi M, Olin CE, Johns HT, et al. Neuroprotective natural antibodies to assemblies of amyloidogenic peptides decrease with normal aging and advancing Alzheimer's disease. Proc Natl Acad Sci U S A. 2009; 106: 12145-12150

34. Marcello A, Wirths O, Schneider-Axmann T, et al. Reduced levels of IgM autoantibodies against N-truncated pyroglutamate Abeta in plasma of patients with Alzheimer's disease. Neurobiol Aging. 2011; 32: 1379-1387

35. Perez-Garmendia R, Gevorkian G. Pyroglutamate-Modified Amyloid Beta Peptides: Emerging Targets for Alzheimer s Disease Immunotherapy. Curr Neuropharmacol. 2013; 11: 491-498

36. Qu BX, Gong Y, Moore C, et al. Beta-amyloid auto-antibodies are reduced in Alzheimer's disease. J Neuroimmunol. 2014; 274: 168-173

37. Moir RD, Tseitlin KA, Soscia S, et al. Autoantibodies to redoxmodified oligomeric Abeta are attenuated in the plasma of Alzheimer's disease patients. J Biol Chem. 2005; 280: 1745817463

38. Gruden MA, Davidova TB, Malisauskas M, et al. Differential neuroimmune markers to the onset of Alzheimer's disease neurodegeneration and dementia: autoantibodies to Abeta((25-35)) oligomers, S100b and neurotransmitters. J Neuroimmunol. 2007; 186: 181-192

39. Millucci L, Ghezzi L, Bernardini G, et al. Conformations and biological activities of amyloid beta peptide 25-35. Curr Protein Pept Sci. 2010; 11: 54-67

40. Weksler ME, Relkin N, Turkenich $\mathrm{R}$, et al. Patients with Alzheimer disease have lower levels of serum anti-amyloid peptide antibodies than healthy elderly individuals. Exp Gerontol. 2002; 37: 943-948

41. Brettschneider S, Morgenthaler NG, Teipel SJ, et al. Decreased serum amyloid beta(1-42) autoantibody levels in Alzheimer's disease, determined by a newly developed immunoprecipitation assay with radiolabeled amyloid beta(1-42) peptide. Biological Psychiatry. 2005; 57: 813-816

42. Nath A, Hall E, Tuzova M, et al. Autoantibodies to amyloid beta-peptide (Abeta) are increased in Alzheimer's disease patients and Abeta antibodies can enhance Abeta neurotoxicity: implications for disease pathogenesis and vaccine development. Neuromolecular Med. 2003; 3: 29-39

43. Du Y, Dodel R, Hampel H, et al. Reduced levels of amyloid betapeptide antibody in Alzheimer disease. Neurology. 2001; 57: 801-805

44. Maftei M, Thurm F, Schnack C, et al. Increased levels of antigen-bound beta-amyloid autoantibodies in serum and cerebrospinal fluid of Alzheimer's disease patients. PLoS One. 2013; 8: e68996

45. Akhtar RS, Licata JP, Luk KC, et al. Measurements of autoantibodies to alpha-synuclein in the serum and cerebral spinal fluids of patients with Parkinson's disease.J Neurochem. 2018,

46. Horvath I, Iashchishyn IA, Forsgren L, et al. Immunochemical Detection of alpha-Synuclein Autoantibodies in Parkinson's Disease: Correlation between Plasma and Cerebrospinal Fluid Levels. ACS Chem Neurosci. 2017; 8: 1170-1176

47. Gruden MA, Sewell RD, Yanamandra K, etal. Immunoprotection against toxic biomarkers is retained during Parkinson's disease progression. J Neuroimmunol. 2011; 233: 221-227

48. Yanamandra K, Gruden MA, Casaite V, et al. alpha-synuclein reactive antibodies as diagnostic biomarkers in blood sera of Parkinson's disease patients. PLoS One. 2011, 6: e18513

49. Shalash A, Salama M, Makar M et al. Elevated Serum alphaSynuclein Autoantibodies in Patients with Parkinson's Disease Relative to Alzheimer's Disease and Controls. Front Neurol. 2017; 8: 720

50. Brudek T, Winge K, Folke J, et al. Autoimmune antibody decline in Parkinson's disease and Multiple System Atrophy; a step towards immunotherapeutic strategies. Mol Neurodegener. $2017 ; 12: 44$

51. Maetzler W, Apel A, Langkamp M, et al. Comparable autoantibody serum levels against amyloid-and inflammationassociated proteins in Parkinson's disease patients and controls. PLoS One. 2014; 9: e88604

52. Smith LM, Schiess MC, Coffey MP, et al. alpha-Synuclein and anti-alpha-synuclein antibodies in Parkinson's disease, atypical Parkinson syndromes, REM sleep behavior disorder, and healthy controls. PLoS One. 2012; 7: e52285

53. Alvarez-Castelao B, Gorostidi A, Ruiz-Martinez J, et al. Epitope Mapping of Antibodies to Alpha-Synuclein in LRRK2 Mutation Carriers, Idiopathic Parkinson Disease Patients, and Healthy Controls. Front Aging Neurosci. 2014; 6: 169 
54. Nagele EP, Han M, Acharya NK, et al. Natural IgG autoantibodies are abundant and ubiquitous in human sera, and their number is influenced by age, gender, and disease. PLoS One. 2013; 8: e60726

55. Petranyi G, Jr Benczur M, Alfoldy P. The effect of single large dose hydrocortisone treatment on IgM and IgG antibody production, morphological distribution of antibody producing cells and immunological memory. Immunology. 1971; 21: 151-158

56. Salinas-Carmona MC, Perez LI, Galan K, et al.
Immunosuppressive drugs have different effect on B lymphocyte subsets and IgM antibody production in immunized BALB/c mice. Autoimmunity. 2009; 42: 537-544

57. Masters CL, Selkoe DJ. Biochemistry of amyloid beta-protein and amyloid deposits in Alzheimer disease. Cold Spring Harb Perspect Med. 2012; 2: a006262

58. Carrillo MC, Blennow K, Soares H, et al. Global standardization measurement of cerebral spinal fluid for Alzheimer's disease: an update from the Alzheimer's Association Global Biomarkers Consortium. Alzheimers Dement. 2013; 9: 137-140 